\title{
Mean field calculations of nucleon-nucleus scattering
}

\author{
R. Crespo, ${ }^{*}$ R. C. Johnson, and J. A. Tostevin \\ Department of Physics, University of Surrey, Guildford, Surrey, GU2 5XH, United Kingdom
}

(Received 8 November 1995)

\begin{abstract}
Corrections to the first order term of the multiple scattering expansion of the nucleon-nucleus optical potential due to the propagation of the projectile nucleon in the target nucleus mean field are estimated. The effects of nonlocalities of the nucleon-nucleon transition amplitude are included. Calculations are performed for nucleon scattering from ${ }^{16} \mathrm{O}$ and ${ }^{208} \mathrm{~Pb}$ at 100,200 , and $400 \mathrm{MeV}$ incident energies. We show that the mean field effect is repulsive and reduces the strength of the local representation of the impulse approximation potential by approximately $25 \%$ in the nuclear interior. [S0556-2813(96)04605-5]
\end{abstract}

PACS number(s): 24.10.Ht, 21.30.Fe, 25.40.Cm

\section{INTRODUCTION}

The nucleon-nucleus optical potential is an important tool finding widespread application in the analysis of nuclear scattering and reaction data. In its own right, nucleonnucleus elastic scattering is a basic reaction from which it is hoped we can identify underlying reaction mechanisms.

The nucleon optical potential is expected to have an energy dependence which arises from a number of different sources. Multiple scattering theories generate a nonlocal expression for the optical potential. Prescriptions which replace this nonlocal expression by an equivalent local potential usually introduce an energy dependence. The free nucleonnucleon $(N N)$ scattering amplitude also has an intrinsic energy dependence as well as being a nonlocal operator. An additional energy dependence arises from exchange effects in the $N N$ amplitude and also from the effects of Pauli blocking in the nuclear medium. Calculations which include all of these effects [1] predict that the real and imaginary central terms of the optical potential have a radial dependence similar to that of the target density. An additional source of energy dependence results from the projectile, propagating between scatterings in the mean field generated by the target nucleons [2]. It is the effect of this mean field that is the subject of the present work.

Phenomenological analyses of nucleon-nucleus (NA) scattering in the energy region from 150 and $400 \mathrm{MeV}$ show [3] that the real central potential deviates considerably from the Fermi distribution shape associated with the target density. The imaginary central term of the optical potential has a volume form. The need for such an unorthodox real central potential shape in this energy region was first noted by Elton [4]. A convincing microscopic theory needs to incorporate an energy dependence which can predict this behavior of the optical potential.

In this work we analyze the energy dependence present in multiple scattering formalisms [2,5]. Detailed calculations of the multiple scattering optical potential have been performed in the absence of the above-mentioned mean field effect. The effects of the nonlocality in the $N N$ transition amplitude on

\footnotetext{
*On leave from Departamento de Física, Instituto Superior Técnico, Lisboa, Portugal.
}

the first order term of the optical potential have been evaluated in detail in $[6]$ and $[7,8]$. The essential difference between these two sets of calculations is the use of starting energy in the evaluation of the $N N$ transition amplitude. A full treatment of this starting energy, which includes the binding of the struck nucleon $[9,10]$, is still needed to assess the importance of off-shell effects associated with the momenta of the interacting nucleon pair. The energy dependence from Pauli blocking effects in the nuclear medium is taken into account by the second order term of the multiple scattering expansion [11]. Local phase-equivalent interactions to the nonlocal Kerman-McManus-Thaler (KMT) optical potential [1] show that these Pauli blocking effects primarily modify the imaginary part of the optical potential in the nuclear interior and that the real and imaginary central terms are essentially of volume shape.

$N N$ information can also be input into nucleon-nucleus calculations through a $g$-matrix effective interaction $g_{01}(\rho)$, appropriate for two nucleons interacting in infinite nuclear matter of density $\rho$ [12]. Such an interaction takes account of Pauli blocking effects within the (infinite) nuclear medium. For intermediate energy protons, the imaginary central term of the resulting nucleon-nucleus optical potential exhibits an essentially volume form. The real part of the potential has a surface-peaked component that becomes increasingly important as the proton incident energy increases, becoming repulsive at incident energies of $400 \mathrm{MeV}$ and above. A qualitative explanation of this phenomenon is presented by Feshbach [13] based on local density ideas. The elastic scattering observables are very sensitive to these features of the $g$-matrix-based calculations. A basic problem of the nuclear matter based $g$-matrix approach is that the local density approximation (LDA) has to be applied in going to the finite nucleus case. The validity of the LDA for the description of the Pauli blocking terms of the microscopic optical potential has been studied recently [14]. An artifact of the approximations made in the LDA is to induce a surface peaking in the imaginary component of the optical potential arising from the Pauli blocking mechanism.

Phenomenological nucleon-nucleus optical potentials have also been deduced from Dirac phenomenological analyses of proton-nucleus elastic scattering data [15]. The deduced Schrödinger-equivalent potentials are found to have real central terms with a characteristic "wine bottle bottom" 
shape and which become repulsive at nucleon incident energies of $400 \mathrm{MeV}$ and above. The potentials have a weak attractive tail whose magnitude decreases as the energy increases. The origin of the wine bottle bottom shape, within this relativistic framework, is the result of delicate cancellations between the large Lorentz scalar and vector components of the relativistic optical potential.

In this work our aim is to shed new light on the origin of this repulsion in the real central component of the optical potential within the nonrelativistic framework. The first order term of the multiple scattering optical potential is evaluated taking into account (i) the mean field generated by the target nucleons on the propagation of the projectile and (ii) the nonlocalities of the $N N$ transition amplitude. To compare the calculations with and without the mean field term we make use of a local representation of the nonlocal optical potentials [14] following the formulation of Horiuchi [16].

\section{MULTIPLE SCATTERING FORMALISMS}

In the multiple scattering approach, the optical potential is expanded in terms of an effective $N N$ transition amplitude. We discuss briefly the multiple scattering formalisms of Watson [2] and KMT [5]. In the KMT formalism the optical potential for elastic scattering is $U=\left\langle\Phi_{0}|\mathscr{C}| \Phi_{0}\right\rangle$ where $\Phi_{0}$ is the target ground state and

$$
\mathscr{U}=(A-1) \tau_{01}\left(E_{0}\right)\left[1+\frac{\mathscr{C}}{\delta} Q_{0} \mathscr{\mathscr { b }}\right]
$$

Here $A$ is the antisymmetrization operator for the $A$ target nucleons and $\delta=E_{0}^{+}-K_{0}-H_{A}$ with $E_{0}\left(=\hbar^{2} k_{0}^{2} / 2 \mu_{N A}\right)$ the proton incident energy in the proton-target $(N A)$ center of mass frame and $\mu_{N A}$ is the $N A$ reduced mass. $H_{A}$ is the internal Hamiltonian of the target, $K_{0}$ the kinetic energy operator of the incident nucleon, and $k_{0}$ is its on-shell momentum. The Pauli blocking operator $Q_{0}$ projects off the target ground state, i.e., $Q_{0}=1-P_{0}$ where $P_{0}=\left|\Phi_{0}\right\rangle\left\langle\Phi_{0}\right|$. The antisymmetrized effective $N N$ transition operator $\tau_{01}\left(E_{0}\right)$ describes the scattering of the projectile from any one of the target nucleons (labeled " 1 "), and satisfies the integral equation

$$
\tau_{01}\left(E_{0}\right)=v_{01}+v_{01} \frac{\mathscr{A}}{\delta} \tau_{01}\left(E_{0}\right)
$$

The effects of the antisymmetrization operator are relevant to the discussion of second and higher order terms only, and means only physical states of the nucleus appear as intermediate states. If the target ground state is a single Slater determinant of occupied single-particle states $|\alpha\rangle$, with singleparticle energies $\epsilon_{\alpha}$, then the first order term of the optical potential can be written $[5,11]$

$$
U^{(1)}=\frac{A-1}{A} \sum_{\alpha}\left\langle\alpha\left|\hat{t}_{01}\left(\hat{\omega}_{\alpha}\right)\right| \alpha\right\rangle .
$$

The sum in $\alpha$ runs over all occupied states and $\hat{\omega}_{\alpha}=E_{0}+\epsilon_{\alpha}$. The corresponding second order term has been discussed elsewhere $[5,11]$. The $N N$ transition operator $\hat{t}_{01}\left(\hat{\omega}_{\alpha}\right)$ is a three-body operator and satisfies the integral equation

$$
\hat{t}_{01}\left(\hat{\omega}_{\alpha}\right)=v_{01}+v_{01} \hat{g}\left(\hat{\omega}_{\alpha}\right) \hat{t}_{01}\left(\hat{\omega}_{\alpha}\right)
$$

where $v_{01}$ is the free $N N$ interaction. The intermediate states propagator is

$$
\hat{g}\left(\hat{\omega}_{\alpha}\right)=\frac{1}{\hat{\omega}_{\alpha}^{+}-K_{0}-K_{1}-V_{1}},
$$

with $K_{1}$ the kinetic energy of the struck nucleon and $V_{1}$ its binding potential to the core of $A-1$ nucleons.

In the impulse approximation, in the treatment of $\hat{g}\left(\hat{\omega}_{\alpha}\right)$, the struck nucleon is assumed free $[7,8]$. Thus, both its binding energy $\epsilon_{\alpha}$ and binding potential $V_{1}$ are neglected. A consideration of these binding effects, which included both the binding energy and binding potential effects, showed them to be small [9]. The three-body operator $\hat{t}_{01}$ is then replaced by the free $N N$ transition amplitude $t_{01}$ that satisfies the integral equation

$$
t_{01}\left(\omega_{0}\right)=v_{01}+v_{01} g\left(\omega_{0}\right) t_{01}\left(\omega_{0}\right)
$$

where the intermediate states propagator is

$$
g\left(\omega_{0}\right)=\frac{1}{\omega_{0}^{+}-K_{01}},
$$

with $K_{01}$ the $N N$ relative motion kinetic energy operator. The energy parameter is now $\omega_{0}=E_{0}-\hbar^{2} \hat{\mathscr{P}}^{2} / 4 m$ with $\hat{\mathscr{P}}$ the momentum operator for the motion of the center of mass (c.m.) of the interacting $N N$ pair [11].

The first order KMT potential is represented diagrammatically in Fig. 1(a). Here the projectile scatters from a target nucleon, assumed free, and the core of $(A-1)$ target nucleons are assumed to remain in their occupied states. In Fig. 1(b) the $N N$ transition amplitude is drawn so as to show explicitly the intermediate $N N$ states in which the active nucleons interact as free particles.

A multiple scattering expansion of the optical potential, in terms of an effective $N N$ transition amplitude $\tau_{W}^{(i)}$, has also been derived by Watson [2]. To first order in $\tau_{W}^{(i)}$,

$$
U^{W}=\left\langle\Phi_{0}\left|\sum_{i} \tau_{W}^{(i)}\right| \Phi_{0}\right\rangle,
$$

where the effective $N N$ amplitude satisfies

$$
\tau_{W}^{(i)}=v_{0 i}+v_{0 i} Q_{0} \frac{1}{\delta-U^{W}} \tau_{W}^{(i)}
$$

In first order all multiple scattering interactions that involve the projectile and the struck nucleon are taken into account. In this formalism the projectile propagates, between scatterings, in the presence of the mean field created by the target nucleons. It is the importance of this mean field on the optical potential and the elastic scattering observables that is the subject of the present work. As discussed in relation to the KMT potential, we will neglect binding effects. Following 

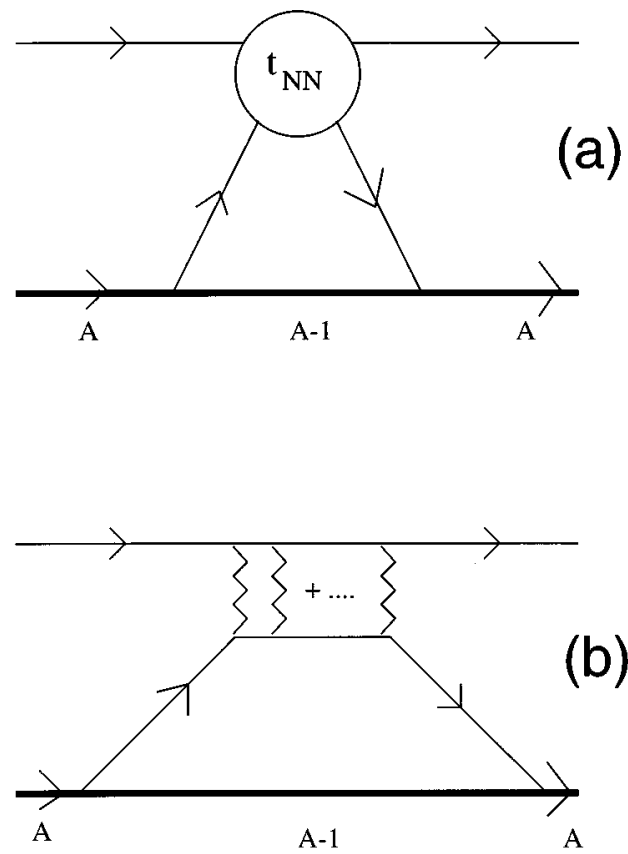

FIG. 1. Diagrammatic representation of the single scattering approximation to the KMT optical potential expansion, indicating (a) the first order $t$-matrix vertex, and (b) showing explicitly the free intermediate states propagation of the active $N N$ pair.

the arguments of Ref. [2], the projection operator $Q_{0}$ in Eq. (9) can also be neglected to the extent that we neglect terms of order $1 / A$. Terms of this order are already neglected in terminating the multiple scattering series to first order. In this limit the optical potential, including the mean field effects, reads

$U^{W}=A\left\langle\Phi_{0}\left|t_{01}(\omega)\right| \Phi_{0}\right\rangle=A\left\langle\Phi_{0}\left|t_{01}\left(\omega_{0}-U^{W}\right)\right| \Phi_{0}\right\rangle$,

represented diagrammatically in Fig. 2, where the projectile now appears "dressed" due to the mean field of the target nucleons. We refer to this model as the Watson or mean field (MF) theory.

\section{LOCAL EQUIVALENT POTENTIAL}

To display the (nonlocal) effects of the mean field in the projectile propagator of the $N N$ amplitude, we will calculate a local equivalent $V_{L}(r)$ to the nonlocal optical potential. The general procedure is as follows. For a nonlocal optical potential $G\left(\vec{r}, \vec{r}^{\prime}\right)$, the Schrödinger equation assumes the form

$$
-\frac{\hbar^{2}}{2 \mu_{N A}} \nabla^{2} \Psi_{\mathrm{NL}}(\vec{r})+\int d \vec{r}^{\prime} G\left(\vec{r}, \vec{r}^{\prime}\right) \Psi_{\mathrm{NL}}\left(\vec{r}^{\prime}\right)=E_{0} \Psi_{\mathrm{NL}}(\vec{r}),
$$

and, if the nonlocal potential satisfies the normal symmetry and rotational invariance requirements, then $G\left(\vec{r}, \vec{r}^{\prime}\right)$

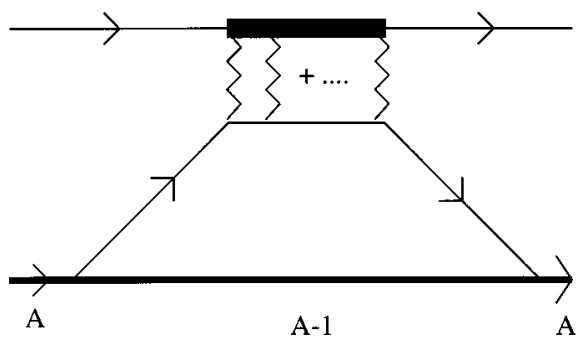

FIG. 2. Diagrammatic representation of the first order term of the mean field optical potential showing the "dressed" intermediate states propagation of the projectile nucleon in the mean field of the core nucleons.

is a function only of the three scalar variables $r^{2}, r^{\prime 2}$, and $\vec{r} \cdot \vec{r}^{\prime} \quad$ or, alternatively, of $\left(\vec{r}+\vec{r}^{\prime}\right)^{2},\left(\vec{r}-\vec{r}^{\prime}\right)^{2}, \quad$ and $\left(\vec{r}+\vec{r}^{\prime}\right) \cdot\left(\vec{r}-\vec{r}^{\prime}\right)$.

Following Horiuchi [16], we define a local Wigner transform [17] potential in terms of the nonlocal potential by

$$
\hat{G}(\vec{r}, \vec{k})=\int d \vec{s} \exp (i \vec{s} \cdot \vec{k}) G(\vec{r}-\vec{s} / 2, \vec{r}+\vec{s} / 2),
$$

and rotational invariance implies that $\hat{G}(\vec{r}, \vec{k})=$ $\hat{G}\left(r^{2}, k^{2},(\vec{r} \cdot \vec{k})^{2}\right)$. The local potential, acting in partial wave $\ell$, is obtained from the Wigner transform upon making the substitutions [16]

$$
k^{2} \rightarrow \frac{2 \mu_{N A}}{\hbar^{2}}\left[E_{0}-V_{L}(r)\right]
$$

$$
(\vec{r} \cdot \vec{k})^{2} \rightarrow \frac{2 \mu_{N A} r^{2}}{\hbar^{2}}\left(E_{0}-V_{L}(r)-\frac{\hbar^{2}(\ell+1 / 2)^{2}}{2 \mu_{N A} r^{2}}\right)
$$

with the result that

$$
\begin{aligned}
V_{L}(r)= & \hat{G}\left(r^{2}, \frac{2 \mu_{N A}}{\hbar^{2}}\left[E_{0}-V_{\mathrm{L}}(r)\right],\right. \\
& \left.\frac{2 \mu_{N A} r^{2}}{\hbar^{2}}\left[E_{0}-V_{L}(r)-\frac{\hbar^{2}(\ell+1 / 2)^{2}}{2 \mu_{N A} r^{2}}\right]\right) .
\end{aligned}
$$

The equivalent potential is in general $\ell$ dependent, which arises from the dependence on the angle between the position $\vec{r}$ and the momentum $\vec{k}$.

\section{TARGET NUCLEUS MODEL}

We will discuss the optical potential for a ${ }^{16} \mathrm{O}$ and ${ }^{208} \mathrm{~Pb}$ target. We assume the target matter densities for ${ }^{16} \mathrm{O}$, in momentum space, to be of the form

$$
\rho(q)=\left[1-\frac{(Z-2)}{6 Z} a^{2} q^{2}\right] \exp \left(-a^{2} q^{2} / 4\right)
$$


with $Z$ the target charge. The range parameter $a$ is taken as $1.77 \mathrm{fm}$ [18]. For ${ }^{208} \mathrm{~Pb}$ we use a two-parameter-Fermidensity distribution [19] with radius parameter $R_{0}=6.624 \mathrm{fm}$ and diffuseness $a=0.549 \mathrm{fm}$.

\section{IMPULSE APPROXIMATION}

The MF theory, Eq. (10), involves the folding of the $N N$ transition amplitude $t_{01}\left(\omega_{0}-U^{W}\right)$ with the target nucleus wave function. The $N N$ energy parameter is $\omega_{0}=E_{0}-\hbar^{2} \hat{\mathscr{P}}^{2} / 4 m$ with $\hat{\mathscr{P}}$ the momentum operator for the motion of the center of mass (c.m.) of the interacting $N N$ pair [11]. In the impulse approximation the potential is not taken into account in the intermediate states propagator, i.e.,

$$
V_{0}=A\left\langle\Phi_{0}\left|t_{01}\left(\omega_{0}\right)\right| \Phi_{0}\right\rangle
$$

In addition, in the evaluation of $\omega_{0}$, we neglect the momentum of the struck nucleon and take the momentum of the projectile to be the incident on-shell value, obtaining

$$
U^{\mathrm{imp}}=A\left\langle\Phi_{0}\left|t_{01}\left(E_{0} / 2\right)\right| \Phi_{0}\right\rangle .
$$

Using the definition of the Wigner transform, Eq. (12), and the results outlined in the Appendix for the momentum space matrix elements of the $N N$ transition amplitude in the target nucleus ground state, then the local representation is

$$
\begin{aligned}
U_{L}^{\mathrm{imp}}(r)= & \frac{A}{(2 \pi)^{3}} \int d \vec{s} \exp (i \vec{s} \cdot \vec{k}) \iint d \vec{q} d \vec{Q} \exp (-i \vec{q} \cdot \vec{r}) \\
& \times \exp (-i \vec{Q} \cdot \vec{s}) \rho(q) \bar{t}_{01}\left(E_{0} / 2, q, Q / 2, \phi_{0}\right),
\end{aligned}
$$

with $\rho(q)$ the target density normalized such that $\rho(0)=1$. Upon carrying out the integral in $\vec{s}$,

$$
U_{L}^{\mathrm{imp}}(r)=A \int d \vec{q} \exp (-i \vec{q} \cdot \vec{r}) \rho(q) \bar{t}_{01}\left(E_{0} / 2, q, Q^{*} / 2, \phi_{0}\right)
$$

where $\bar{t}_{01}$ is to be evaluated at effective momentum $Q^{*}$ given by

$$
Q^{* 2}=\frac{2 \mu_{N A}}{\hbar^{2}}\left[E_{0}-U_{L}^{\mathrm{imp}}(r)\right]
$$

Equation (19) is evaluated by expanding $Q^{*}$ to first order in $U_{L}^{\text {imp }}(r)$ about $Q_{0}=\sqrt{2 \mu_{N A} E_{0} / \hbar^{2}}$, i.e.,

$$
U_{L}^{\mathrm{imp}}(r)=U_{0}\left(E_{0} / 2, r\right)+U_{L}^{\mathrm{imp}}(r) \Delta \mathrm{imp}(r),
$$

where

$$
\begin{aligned}
\Delta^{\mathrm{imp}}(r)= & -A\left(\frac{\mu_{N A}}{2 \hbar^{2} E_{0}}\right)^{1 / 2} \int d \vec{q} \exp (-i \vec{q} \cdot \vec{r}) \rho(q) \\
& \times\left[\frac{\partial}{\partial Q} \bar{t}_{01}\left(E_{0} / 2, q, Q / 2, \phi_{0}\right)\right]_{Q=Q_{0}} .
\end{aligned}
$$

It follows that

$$
U_{L}^{\mathrm{imp}}(r)=\mathscr{F}^{\mathrm{imp}}\left(E_{0}, r\right) U_{0}\left(E_{0} / 2, r\right)
$$

$$
=\left[1-\Delta^{\mathrm{imp}}(r)\right]^{-1} U_{0}\left(E_{0} / 2, r\right),
$$

where $U_{0}(\mathscr{E}, r)$ is a local, energy-dependent potential,

$$
\begin{aligned}
U_{0}(\mathscr{E}, r)= & A \int d \vec{q} \exp (-i \vec{q} \cdot \vec{r}) \rho(q) \overline{t_{01}} \\
& \times\left(\mathscr{E}, q,\left[\mu_{N A} \mathscr{E} / \hbar^{2}\right]^{1 / 2}, \phi_{0}\right) .
\end{aligned}
$$

In the impulse approximation optical potential, Eq. (23), the factor $\Delta^{\mathrm{imp}}$ arises from the nonlocality of the free $N N$ transition operator. If the latter were local, the matrix element of $\bar{t}_{01}$ entering Equation (19) would depend only on $q$ and not $Q$. Equation (22) then gives $\Delta^{\mathrm{imp}}=0$.

\section{MEAN FIELD OPTICAL POTENTIAL}

\section{A. Local potential representation}

In the MF theory, due to the presence of the potential in the intermediate states propagator, we have

$U^{W}=A\left\langle\Phi_{0}\left|t_{01}(\omega)\right| \Phi_{0}\right\rangle=A\left\langle\Phi_{0}\left|t_{01}\left(\omega_{0}-U^{W}\right)\right| \Phi_{0}\right\rangle$.

For the purpose of solving the implicit equation for $U^{W}$ we will assume that $U^{W}$ in the $N N$ propagator is independent of the position of the projectile nucleon in the nucleus. This is clearly an approximation which will be best in the nuclear interior of a heavy nucleus. Expanding the $N N$ amplitude about the energy parameter $\omega_{0}$, then

$$
\begin{aligned}
U^{W} & =A\left\langle\Phi_{0}\left|t_{01}\left(\omega_{0}\right)\right| \Phi_{0}\right\rangle-U^{W} A\left\langle\Phi_{0}\left|t_{01}^{\prime}\left(\omega_{0}\right)\right| \Phi_{0}\right\rangle+\cdots \\
& =V_{0}-U^{W} V_{0}^{\prime}+\cdots
\end{aligned}
$$

with

$$
V_{0}=A\left\langle\Phi_{0}\left|t_{01}\left(\omega_{0}\right)\right| \Phi_{0}\right\rangle, \quad V_{0}^{\prime}=A\left\langle\Phi_{0}\left|t_{01}^{\prime}\left(\omega_{0}\right)\right| \Phi_{0}\right\rangle,
$$

and where $t_{01}^{\prime}$ denotes the differential of $t_{01}$ with respect to the energy parameter, i.e.,

$$
t_{01}^{\prime}\left(\omega_{0}\right)=\left.\frac{\partial t_{01}(\omega)}{\partial \omega}\right|_{\omega=\omega_{0}} .
$$

Comparing $U^{W}$ and $V_{0}$, the leading MF correction term $U^{W} V_{0}^{\prime}$ involves a product of the foldings of the $N N$ amplitude and its energy derivative in the target ground state.

The (local) Wigner transform $U_{L}^{W}$ of the (nonlocal) $U^{W}$ is therefore written

$$
U_{L}^{W}=\hat{V}_{0}-U^{W} \hat{V}_{0}^{\prime}+\cdots
$$

where the carets denote the Wigner transforms of $V_{0}$ and $V_{0}^{\prime}$. For the purpose of estimating the mean field effects, we evaluate the local representation $U_{L}^{W}$, Eq. (29), of the MF potential by replacing

$$
U_{L}^{W} \approx \hat{V}_{0}-U_{L}^{W} \hat{V}_{0}^{\prime}+\cdots
$$

Using the definition of the Wigner transform, Eq. (12), and the results in the Appendix, then 


$$
\begin{aligned}
U_{L}^{W}(r)= & \frac{A}{(2 \pi)^{3}} \int d \vec{s} \exp (\overrightarrow{i s} \cdot \vec{k}) \iint d \vec{q} d \vec{Q} \exp (-i \vec{q} \cdot \vec{r}) \\
& \times \exp (-i \vec{Q} \cdot \vec{s}) \rho(q)\left[\bar{t}_{01}\left(\omega_{0}, q, Q / 2, \phi_{0}\right)\right. \\
& \left.-U_{L}^{W}(r) \vec{t}_{01}\left(\omega_{0}, q, Q / 2, \phi_{0}\right)+\cdots\right] .
\end{aligned}
$$

Evaluating the integral in $\vec{s}$, and resumming the expansion, this can be rewritten as

$$
U_{L}^{W}(r)=A \int d \vec{q} \exp (-i \vec{q} \cdot \vec{r}) \rho(q) \bar{t}_{01}\left(\omega^{*}, q, Q^{*} / 2, \phi_{0}\right)
$$

with an effective energy parameter $\omega^{*}$ and momentum $Q^{*}$ given by

$$
\begin{gathered}
\omega^{*}=E_{0}-U_{L}^{W}(r)-\hbar^{2} Q^{* 2} / 4 m, \\
Q^{* 2}=\frac{2 \mu_{N A}}{\hbar^{2}}\left[E_{0}-U_{L}^{W}(r)\right],
\end{gathered}
$$

or, assuming that $\mu_{N A} \approx m$,

$$
\begin{gathered}
\omega^{*}=E_{0}-U_{L}^{W}(r)-\hbar^{2} Q^{* 2} / 4 m=\left[E_{0}-U_{L}^{W}(r)\right] / 2, \\
Q^{*}=\left[\frac{2 m}{\hbar^{2}}\left[E_{0}-U_{L}^{W}(r)\right]\right]^{1 / 2}=2\left[\frac{m \omega^{*}}{\hbar^{2}}\right]^{1 / 2} .
\end{gathered}
$$

With $\omega^{*}$ given by Eq. (34), we note that

$$
U_{L}^{W}(r)=U_{0}\left(\omega^{*}, r\right)
$$

with $U_{0}$ given by Eq. (24). Expanding the potential $U_{0}$, to first order about the on-shell energy $E_{0} / 2$, the local equivalent potential reads

$$
\begin{aligned}
U_{L}^{W}(r) & =U_{0}\left(E_{0} / 2, r\right)+U_{L}^{W}(r) \Delta^{W}(r) \\
& =\mathscr{F}^{W}\left(E_{0}, r\right) U_{0}\left(E_{0} / 2, r\right),
\end{aligned}
$$

with

$$
\begin{gathered}
\Delta^{W}(r)=-\left.\frac{1}{2} \frac{d U_{0}(\mathscr{E}, r)}{d \mathscr{E}}\right|_{\mathscr{E}=E_{0} / 2}, \\
\mathscr{F}^{W}\left(E_{0}, r\right)=\left[1-\Delta^{W}(r)\right]^{-1} .
\end{gathered}
$$

Equations (37) and (38) should be compared with Eqs. (22), (23), and (24). Both involve corrections to the potential $U_{0}\left(E_{0} / 2, r\right)$. The factor $\mathscr{F}^{W}$ includes effects due to the mean field in the $N N$ propagator as well as the nonlocal effects included in $\mathscr{F}^{\text {imp }}$. Different partial derivatives of the $N N$ transition amplitude are involved in the two cases.

The numerical calculations of the MF optical potential presented in the following sections are obtained by direct evaluation of Eq. (24) at $\mathscr{E}=E_{0} / 2$ and neighboring energies to calculate $U_{0}\left(E_{0} / 2, r\right)$ and its energy derivative. We note, from Eqs. (37) and (23), that the mean field corrections to the impulse approximation can now be written

$$
U_{L}^{W}(r)=\mathscr{G}\left(E_{0}, r\right) U_{L}^{\mathrm{imp}}(r),
$$
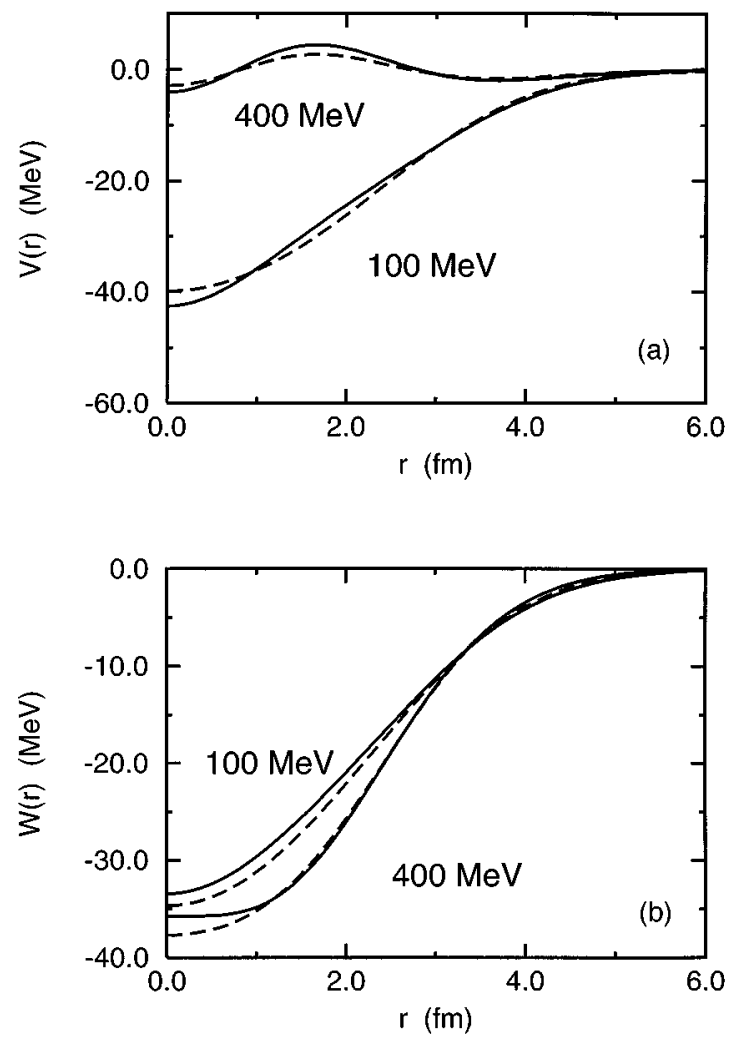

FIG. 3. Calculated real (a) and imaginary (b) central terms of $U_{0}\left(E_{0} / 2, r\right)$ (solid curves), and the on-shell, Eq. (41), (dashed curves) optical potentials at 100 and $400 \mathrm{MeV}$ nucleon energy.

with $\mathscr{G}\left(E_{0}, r\right)=\mathscr{F}^{W}\left(E_{0}, r\right) / \mathscr{F}^{\mathrm{imp}}\left(E_{0}, r\right)$. Some general features of the potential are discussed below.

\section{B. Qualitative features of the optical potential}

We anticipate that the shapes of the local potentials $U_{L}^{W}(r)$ and $U_{L}^{\mathrm{imp}}(r)$ are primarily determined by $U_{0}\left(E_{0} / 2, r\right)$, with modifications in the nuclear interior due to the multipliers $\mathscr{F}^{W}$ and $\mathscr{F}^{\text {imp }}$. The magnitude of $\mathscr{F}^{W}\left(E_{0}, r\right)$ is essentially determined by the energy derivative of the $N N$ transition amplitude and, for the purpose of estimating this effect, we can take the $N N$ transition amplitude on shell. It is well known that, on shell, the imaginary part of the spin-isospin-averaged central term of the $N N$ amplitude, $\bar{t}_{01}$, varies only slowly as a function of energy. The real part is negative and its magnitude decreases somewhat more rapidly with energy, the scattering amplitude becoming mainly absorptive. We expect therefore that the energy derivative of $U_{0}(\mathscr{E}, r)$ will have a positive real component and hence that the function $\mathscr{F}^{W}\left(E_{0}, r\right)$ will take the form of a real suppression factor in the nuclear interior.

Since the nonlocal effects due to the $N N$ operator and the mean field have been expressed as corrections $\mathscr{F}^{W}$ and $\mathscr{F}^{\text {imp }}$ to the local interaction $U_{0}\left(E_{0} / 2, r\right)$, it is useful to clarify the nature of this potential. If the active $N N$ pair interacts on the energy shell, then (see Appendix)

$$
Q^{2}+q^{2} / 4=k_{0}^{2},
$$

and hence we can define the on-shell local potential as 

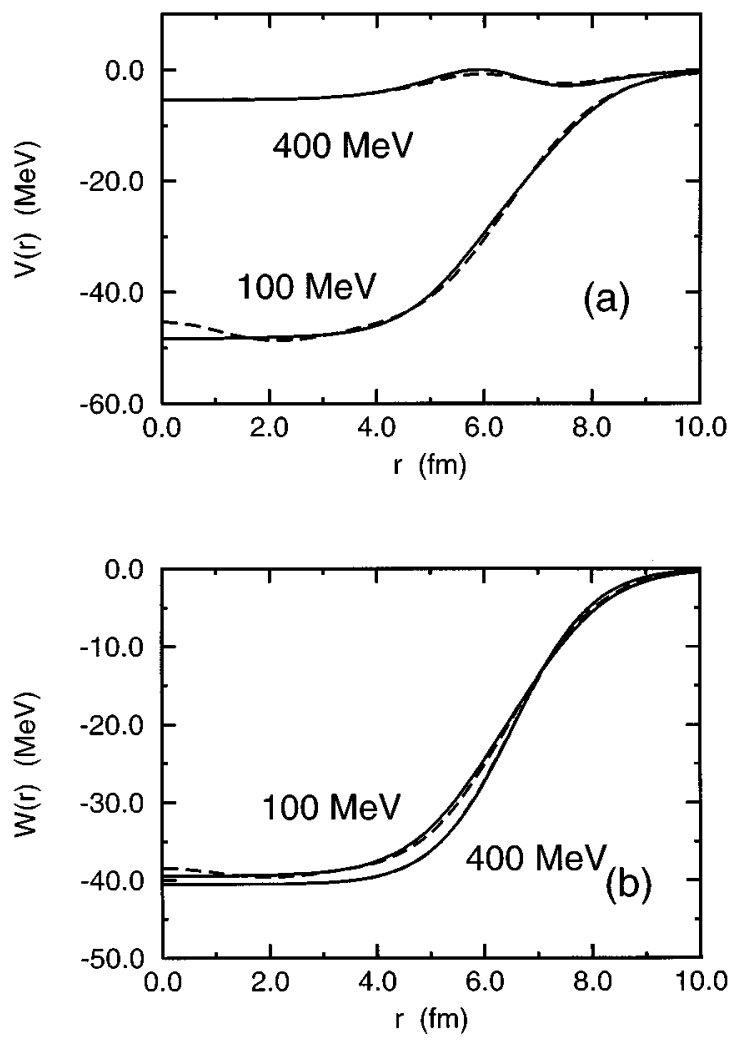

FIG. 4. As for Fig. 3 but for the nucleon- ${ }^{208} \mathrm{~Pb}$ system.

$$
\begin{aligned}
U_{\text {on }}(r)= & A \int d \vec{q} \exp (-i \vec{q} \cdot \vec{r}) \\
& \times \rho(q) \bar{t}_{01}\left(E_{0} / 2, q,\left[k_{0}^{2}-q^{2} / 4\right]^{1 / 2} / 2, \phi_{0}\right) .
\end{aligned}
$$

It follows that at $q=0, Q=k_{0}$, the on-shell potential will coincide with $U_{0}\left(E_{0} / 2, r\right)$ and, in the zero range limit for $t_{01}, U_{0}\left(E_{0} / 2, r\right)=U_{\text {on }}(r)$. We see, however, that for a realistic finite-ranged $N N$ amplitude and nonzero momentum transfers the calculation of $U_{0}\left(E_{0} / 2, r\right)$ requires knowledge of the $N N$ transition amplitude off the energy shell. The potentials $U_{0}\left(E_{0} / 2, r\right)$ and $U_{\text {on }}(r)$ are thus expected to differ at the nuclear surface due to the finite range of the $N N$ interaction. We note, however, that at high incident energies the on-shell potential might be expected to provide a reasonable representation of $U_{0}\left(E_{0} / 2, r\right)$ to the extent that $\left[k_{0}^{2}-q^{2} / 4\right] \rightarrow k_{0}^{2}$ in Eq. (41) in this limit. In addition, for heavier targets with short-ranged momentum space density distributions $U_{0}\left(E_{0} / 2, r\right)$ is expected to be well described by the on-shell interaction $U_{\text {on }}(r)$ in the intermediate energy region.

In Fig. 3 we compare the calculated real (a) and imaginary (b) central terms of $U_{0}$ (solid curves) and the on-shell, Eq. (41) (dashed curves), optical potentials for nucleon scattering from ${ }^{16} \mathrm{O}$ at 100 and $400 \mathrm{MeV}$. Figure 4 shows the corresponding calculations for a ${ }^{208} \mathrm{~Pb}$ target. As discussed above, $U_{0}$ and the on-shell interaction are essentially indistinguishable in this energy range, particularly for the heavier target.
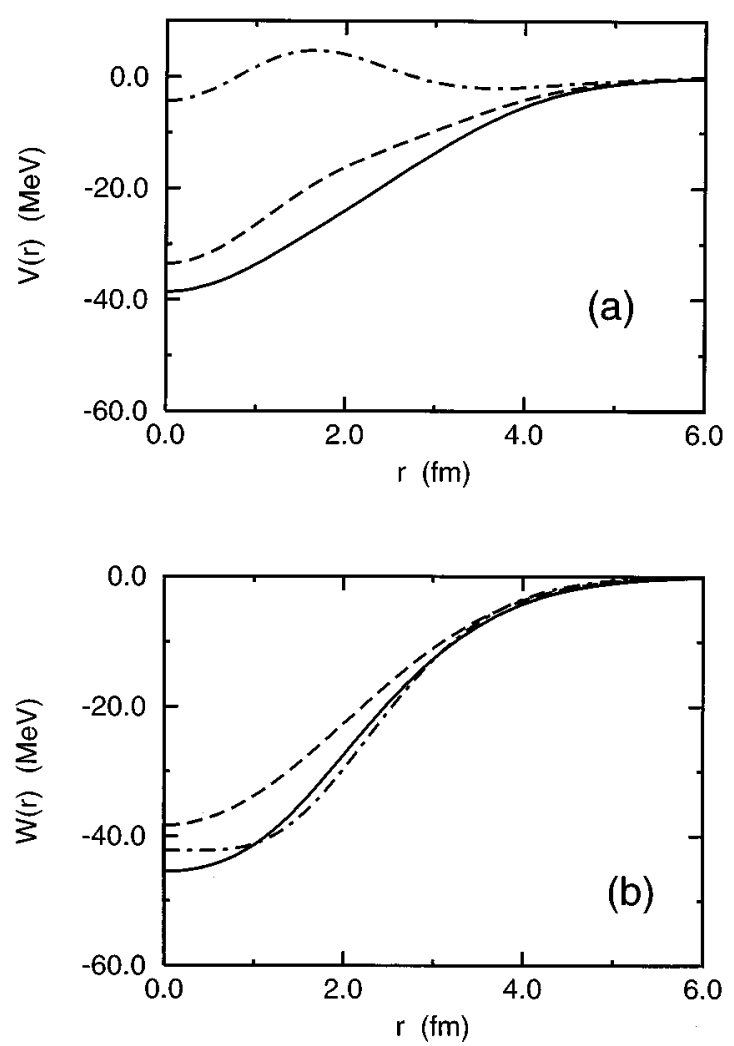

FIG. 5. Calculated real (a) and imaginary (b) central terms of the Wigner transform of the impulse approximation interaction for nucleon- ${ }^{16} \mathrm{O}$ scattering in the absence of the Coulomb interaction. The potentials at 100,200 , and $400 \mathrm{MeV}$ are shown by the solid, dashed, and dash-dotted curves, respectively.

\section{NUMERICAL RESULTS}

\section{A. $N N$ scattering amplitude}

In all calculations the $N N$ scattering amplitudes are calculated exactly, both on and off the energy shell, from the Paris [20,21] $N N$ potential. What is actually required for the mean field calculations is the spin-isospin-averaged central Wolfenstein amplitude $\mathscr{C}_{0}\left(E_{0} / 2, q, k_{0} / 2, \phi_{0}\right)$ for the calculation of $U_{0}\left(E_{0} / 2, r\right)$ and the on-shell variant $\mathscr{C}_{0}\left(E_{0} / 2, q,\left[k_{0}^{2}-q^{2} / 4\right]^{1 / 2} / 2, \phi_{0}\right)$ for the calculation of $U_{\text {on }}(r)$, above.

\section{B. Energy dependence of the potentials}

In Fig. 5 we present the real (a) and imaginary (b) central terms of the local Wigner transform (WT) approximation $U_{L}^{\text {imp }}$ to the impulse approximation interaction, Eq. (23), for the nucleon- ${ }^{16} \mathrm{O}$ system, in the absence of the Coulomb interaction. The potentials at 100, 200, and $400 \mathrm{MeV}$ incident energy are presented by the solid, dashed, and dash-dotted curves, respectively. We note that on this energy interval the real central term of the impulse approximation potential has an essentially volume shape at $200 \mathrm{MeV}$, developing the "wine bottle bottom" shape at the higher energy. The real potential becomes repulsive at $400 \mathrm{MeV}$ and above. The volume form for the impulse approximation interaction at 200 $\mathrm{MeV}$ is contrary to the expectations of phenomenological analyses which show evidence of a wine-bottle-bottom- 

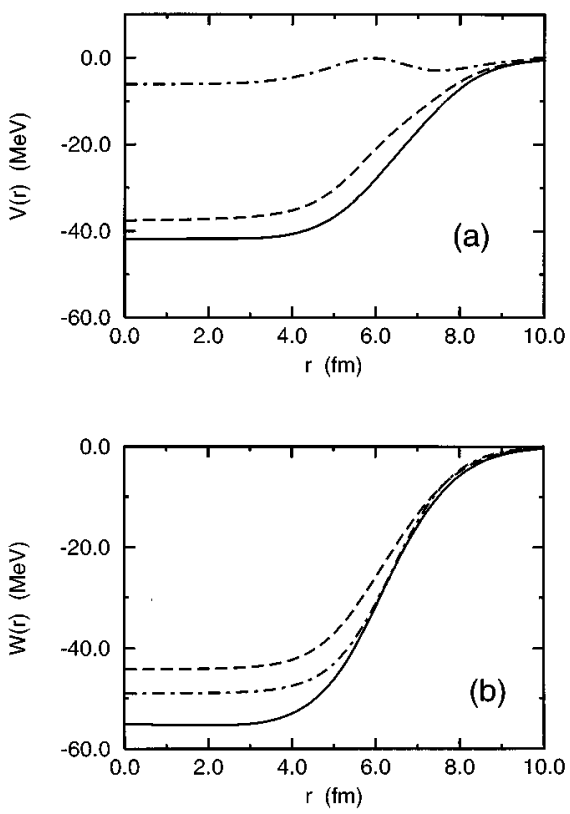

FIG. 6. As for Fig. 5 but for the nucleon- ${ }^{208} \mathrm{~Pb}$ system.

shaped central term even at the lower of these energies [3]. This effect appears not to be correctly reproduced by the impulse approximation optical potential. Figure 6 shows the results for a ${ }^{208} \mathrm{~Pb}$ target, with qualitatively similar results.

Before showing the results for the mean field interaction, we clarify the nature of the modulating function $\mathscr{F}^{W}\left(E_{0}, r\right)$ which multiplies $U_{0}\left(E_{0} / 2, r\right)$ in the local mean field interaction of Eq. (37), and the mean field correction factor $\mathscr{G}\left(E_{0}, r\right)$ which multiplies the WT of the impulse approximation interaction in Eq. (39). Figures 7(a) and 7(b) show the real (solid curves) and imaginary (dashed curves) parts of the calculated $\mathscr{F}^{W}\left(E_{0}, r\right)$ for the nucleon- ${ }^{16} \mathrm{O}$ and ${ }^{208} \mathrm{~Pb}$ systems, respectively, at $E_{0}=100,200$, and $400 \mathrm{MeV}$ incident energies. What is actually plotted is $1-\mathscr{F}^{W}\left(E_{0}, r\right)$. As expected from the earlier qualitative discussion, the $\mathscr{F}^{W}$ are essentially real. Their effect is to reduce $U_{0}\left(E_{0} / 2, r\right)$ by an order of $10 \%$ in the nuclear interior. At $E_{0}=100 \mathrm{MeV}$, the imaginary part becomes significant due to the increase of the energy derivative of the $N N$ transition amplitude.

Parts (a) and (b) of Fig. 8 show the real (solid curves) and imaginary (dashed curves) parts of the calculated $\mathscr{G}\left(E_{0}, r\right)$, presented as $1-\mathscr{G}\left(E_{0}, r\right)$, for the nucleon- ${ }^{16} \mathrm{O}$ and ${ }^{208} \mathrm{~Pb}$ system, respectively, at $E_{0}=100,200$, and $400 \mathrm{MeV}$ incident energy. We note that the strength of the real part of the correction term is essentially constant with energy and dominates the imaginary part at energies of $200 \mathrm{MeV}$ and above. $\mathscr{G}\left(E_{0}, r\right)$, the mean field correction factor to the impulse approximation, is such as to reduce the strength of the impulse approximation potentials by approximately $25 \%$ in the nuclear interior. These corrections fall rapidly at the nuclear surface.

In the following, we present the local mean field interactions without further reference to $\mathscr{F}^{W}$ or $\mathscr{G}$. We note, however, that the range of these modifications is essentially that of the target density. In fact, for heavier systems with short range momentum space density distributions, we can, to a good approximation, replace the $N N$ transition amplitude in Eq. (24) by that at zero momentum transfer. It then follows
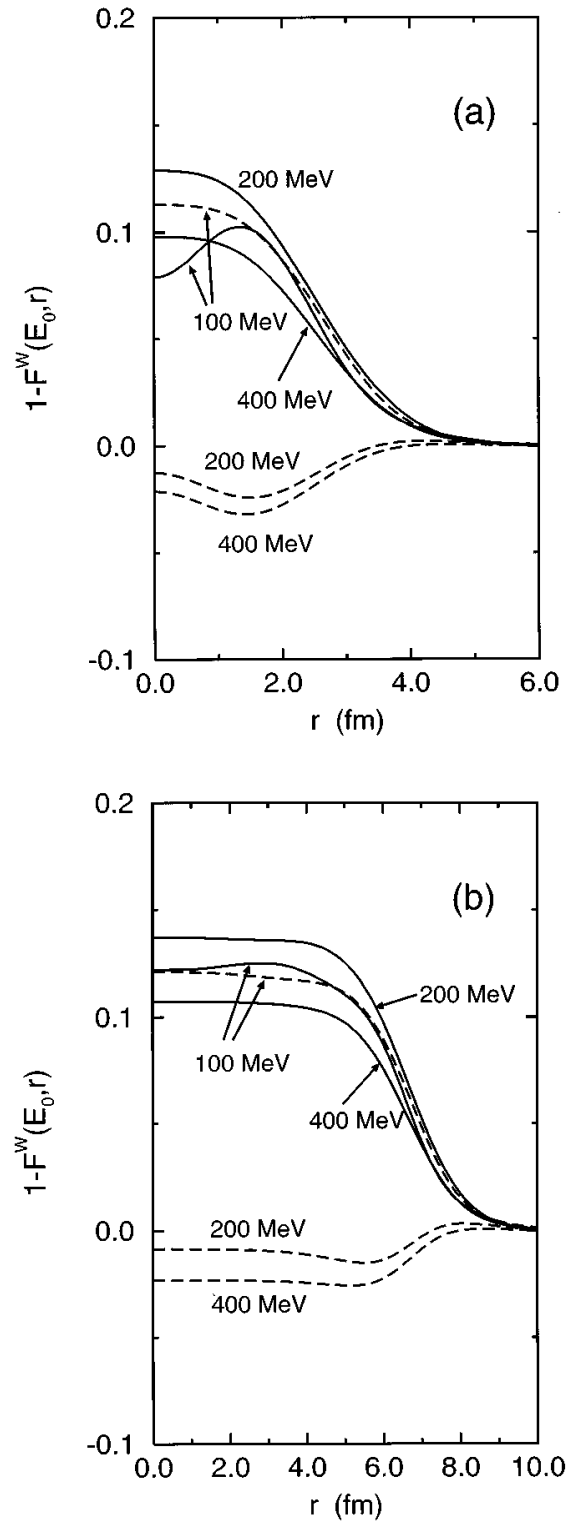

FIG. 7. Calculated real (solid curves) and imaginary (dashed curves) parts of $1-\mathscr{F}^{W}\left(E_{0}, r\right)$ for (a) the nucleon- ${ }^{16} \mathrm{O}$ and (b) the nucleon- ${ }^{208} \mathrm{~Pb}$ system at incident energies $E_{0}=100,200$, and 400 $\mathrm{MeV}$.

that the radial distribution of $\mathscr{F}^{W}$ in Eq. (38) is that of the target nucleus density. Thus, while Pauli blocking medium effects follow the square of the target density and modify the impulse approximation potential in the nuclear interior $[1,11]$ the present mean field effect estimates essentially follow the target density.

Figure 9 shows the calculated real (a) and imaginary (b) central terms of the mean field optical potential, given by Eq. (37), for nucleon scattering from ${ }^{16} \mathrm{O}$. The potentials at 100 , 200, and $400 \mathrm{MeV}$ incident energy are presented by the solid, dashed, and dash-dotted curves, respectively. Figure 10 shows the results for scattering from ${ }^{208} \mathrm{~Pb}$. As was clarified by reference to the $\mathscr{G}\left(E_{0}, r\right)$ factors in Fig. 8, the mean field effects have reduced the central terms of the optical potential in the nuclear volume with respect to those of the WT (local) equivalent for the impulse approximation interactions, Figs. 5 and 6. 

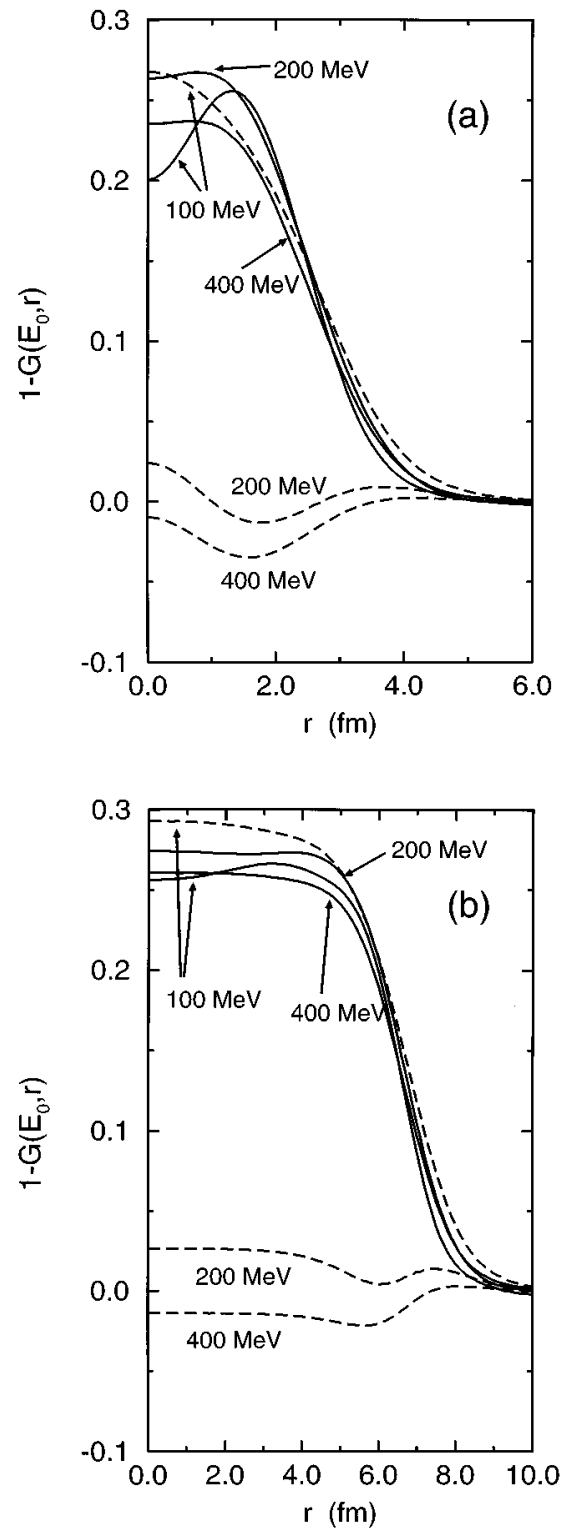

FIG. 8. Calculated real (solid curves) and imaginary (dashed curves) parts of $1-\mathscr{G}\left(E_{0}, r\right)$ for (a) the nucleon- ${ }^{16} \mathrm{O}$ and (b) the nucleon- ${ }^{208} \mathrm{~Pb}$ system at incident energies $E_{0}=100,200$, and 400 $\mathrm{MeV}$.

We have chosen to represent the nonlocal effects associated with the projectile nucleon moving in the nuclear mean field by calculating Wigner transform local equivalents to the nonlocal mean field and impulse approximation interactions. We will not present calculations of scattering observables for two reasons. First, while the Wigner transform local representations are a useful approximation for evaluating and assessing the modifications induced by the mean field, the numerical potentials do not agree precisely with those derived from an inversion analysis [22] of the exact (momentum space) impulse approximation calculations. Second, in estimating the mean field effects we have assumed, in solving the implicit Eq. (25) for $U^{W}$, that $U^{W}$ is independent of the position of the projectile nucleon in the nucleus. This approximation clearly favors the nuclear interior of a heavy nucleus, a region to which elastic scattering observables are
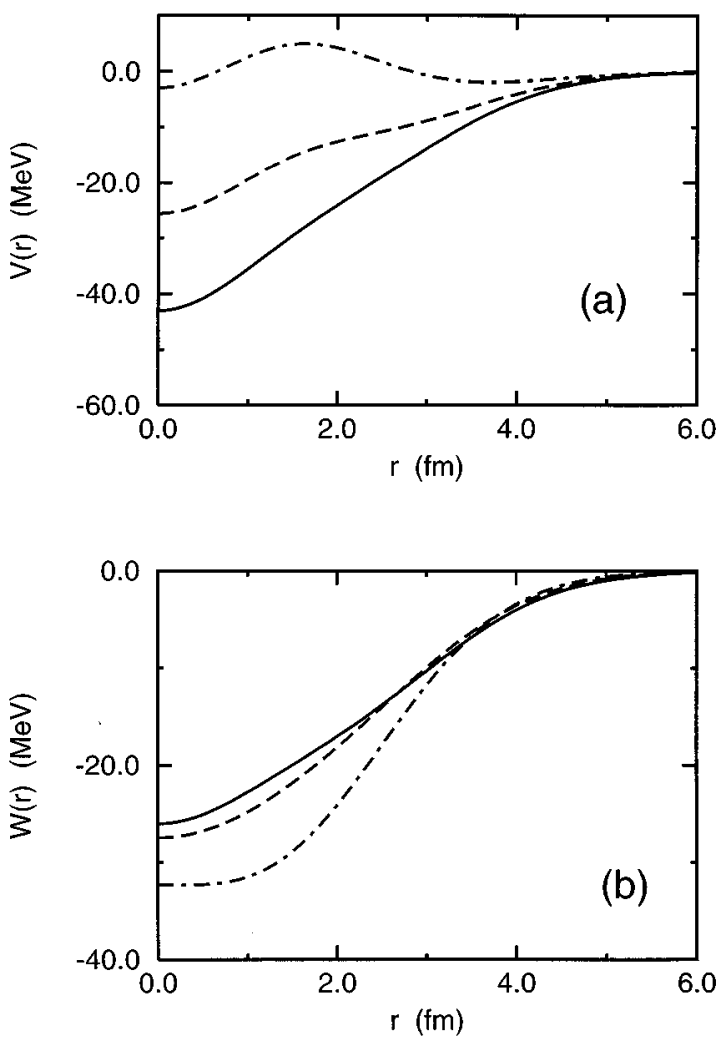

FIG. 9. Calculated real (a) and imaginary (b) central terms of the mean field optical potential, given by Eq. (37), for proton scattering from ${ }^{16} \mathrm{O}$. The potentials at 100,200 , and $400 \mathrm{MeV}$ incident energy are presented by the solid, dashed, and dash-dotted curves, respectively.

relatively insensitive. This latter approximation results in the calculated mean field corrections to the optical potential essentially following the target density and thus the surface behavior of the mean field interaction is not well determined in the present work. The calculated elastic cross section angular distributions do not show particular sensitivity to the mean field corrections to the central potentials, which were supplemented by a spin-orbit interaction derived from the inversion analysis of the exact momentum space calculations. The calculated vector analyzing powers do show some sensitivity to the changes, producing a deeper first minimum at $200 \mathrm{MeV}$, a trend shared by the experimental data.

\section{CONCLUDING REMARKS}

Our aim in this work was to clarify the origin of additional repulsion in the real central component of the optical potential within the nonrelativistic framework. We have estimated the corrections to the first order term of the multiple scattering expansion of the optical potential due to the effects of the mean field generated by the target nucleons on the propagation of the projectile nucleon. The nonlocalities of the $N N$ transition amplitude are included. Calculations are presented for nucleon scattering from ${ }^{16} \mathrm{O}$ and ${ }^{208} \mathrm{~Pb}$ at 100 , 200, and $400 \mathrm{MeV}$ incident energy.

We have chosen to represent the nonlocal effects by calculating Wigner transform local equivalents to the nonlocal 

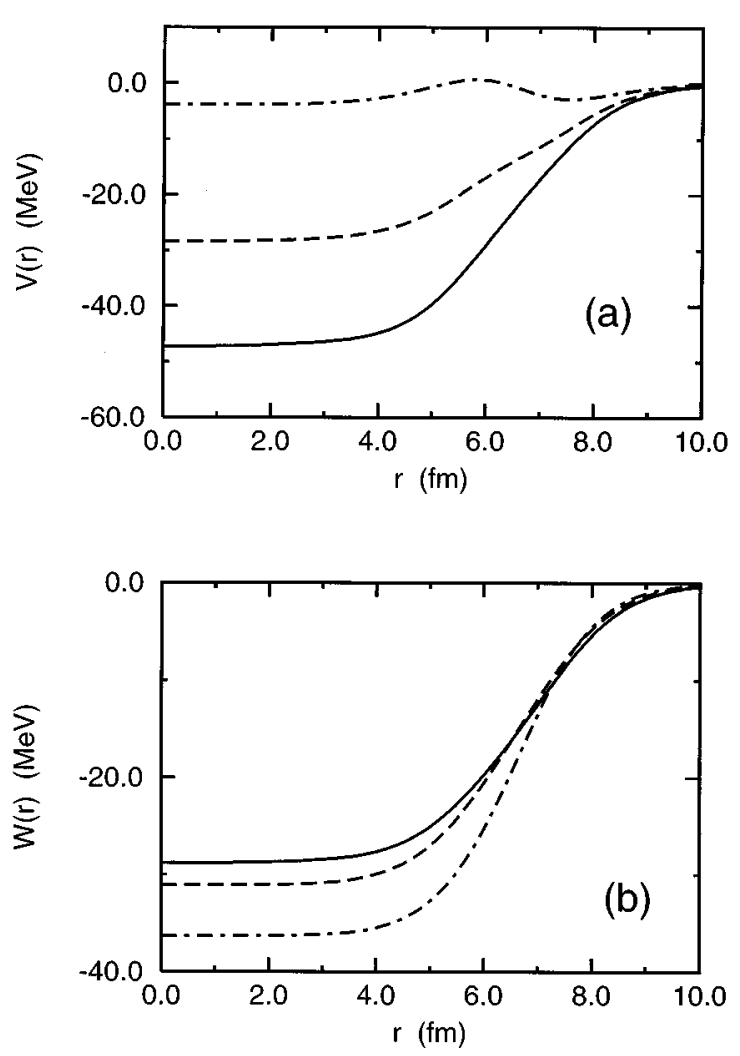

FIG. 10. As for Fig. 9 but for the nucleon- ${ }^{208} \mathrm{~Pb}$ system.

mean field and impulse approximation optical interactions. We have shown that real central terms of the local (Wigner transform) representation of the impulse approximation optical potential are attractive at energies in the region of 200 $\mathrm{MeV}$, becoming repulsive at $400 \mathrm{MeV}$ and above. Our estimate of the mean field effects is approximate and does not provide a realistic description of the associated corrections in the nuclear surface. We do not therefore compare our predictions with scattering data. Our calculated mean field effects essentially follow the target nucleus density and are most reliable at the nuclear center. They are found to reduce the strength of the impulse approximation interactions by $25 \%$ in the nuclear volume, providing an additional repulsive effect at low energies.

\section{ACKNOWLEDGMENTS}

The financial support of the United Kingdom Engineering and Physical Sciences Research Council (EPSRC) in the form of Grants Nos. GR/J95867, GR/K33026, and GR/ H89678, and of JNICT (Portugal), Programa Praxis XXI BPD/4129/94, is gratefully acknowledged.

\section{APPENDIX}

The matrix elements of the $N N$ transition amplitude in the target nucleus ground state are, in momentum space,

$$
\begin{aligned}
\left\langle\vec{k} \Phi_{0}\left|t_{01}\left(\omega_{0}\right)\right| \Phi_{0} \vec{k}\right\rangle= & \frac{1}{A} \sum_{\alpha}\left\langle\vec{k}^{\prime} \alpha\right| t_{01}\left(\omega_{0}|\vec{k} \alpha\rangle\right. \\
= & \frac{1}{A} \sum_{\alpha} \int d^{3} P\langle\alpha \mid \vec{P}-\vec{q} / 2\rangle \\
& \times\left\langle\vec{k}^{\prime}\left|t_{01}\left(\omega_{0}\right)\right| \vec{k}\right\rangle\langle\vec{P}+\vec{q} / 2 \mid \alpha\rangle,
\end{aligned}
$$

with $\vec{P}=\left(\vec{p}+\vec{p}^{\prime}\right) / 2$ and $\vec{Q}=\left(\vec{k}+\vec{k}^{\prime}\right) / 2$ the mean values of the struck and scattered nucleon momenta,

$$
\vec{\kappa}=\frac{1}{2}(\vec{k}-\vec{P}-\vec{q} / 2), \quad \vec{\kappa}^{\prime}=\frac{1}{2}\left(\vec{k}^{\prime}-\vec{P}+\vec{q} / 2\right),
$$

and the energy parameter is

$$
\omega_{0}=E_{0}-\frac{\hbar^{2}}{4 m}(\vec{Q}+\vec{P})^{2} .
$$

The product of target single-particle wave functions is strongly peaked about $P=0$. Thus, the potential matrix elements sample the $N N$ amplitude at and near $\vec{P}=0$. The smooth variation of the $N N$ amplitude over the range of relevant momenta leads to the optimal factorization form of the optical potential and, for a closed shell nucleus [7,8],

$$
\begin{aligned}
\left\langle\vec{k}^{\prime} \Phi_{0}\left|t_{01}\left(\omega_{0}\right)\right| \Phi_{0} \vec{k}\right\rangle= & \rho(q)\left\langle\frac{1}{2}\left(\vec{k}^{\prime}+\vec{q} / 2\right)\right| \\
& \times \overline{t_{01}}\left(E_{0}-\hbar^{2} Q^{2} / 4 m\right)\left|\frac{1}{2}(\vec{k}-\vec{q} / 2)\right\rangle
\end{aligned}
$$

with $\bar{t}_{01}$ the spin-isospin average of the $N N$ transition amplitude. $\rho(q)$ is the Fourier transform of the target density, normalized to $\rho(0)=1$. Reexpressing the $N N$ amplitude

$$
\left\langle\vec{\kappa}^{\prime}\left|\bar{t}_{01}\left(\omega_{0}\right)\right| \vec{\kappa}\right\rangle=\bar{t}_{01}\left(\omega_{0}, \vec{\kappa}^{\prime}, \vec{\kappa}\right)=\bar{t}_{01}\left(\omega_{0}, \vec{q}, \vec{Q}\right),
$$

with $\vec{q}=\left(\vec{\kappa}^{\prime}-\vec{\kappa}\right)$ the momentum transfer and $\vec{Q}=\left(\vec{\kappa}^{\prime}+\vec{\kappa}\right) / 2$ the total $N N$ momentum. The momentum space matrix elements are therefore

$$
\begin{aligned}
\left\langle\vec{k}^{\prime} \Phi_{0}\left|t_{01}\left(\omega_{0}\right)\right| \Phi_{0} \vec{k}\right\rangle & =\rho(q) \overline{t_{01}}\left(E_{0}-\hbar^{2} Q^{2} / 4 m, \vec{q}, \vec{Q} / 2\right) \\
& =\rho(q) \overline{t_{01}}\left(E_{0}-\hbar^{2} Q^{2} / 4 m, q, Q / 2, \phi\right),
\end{aligned}
$$

where $\phi$ is the angle between vectors $\vec{Q}$ and $\vec{q}$. Systematic studies of the $N N$ transition amplitude have shown that the central and spin-orbit components depend only very weakly upon the angle $\phi[21]$. We thus calculate the $N N$ amplitude using the on-shell value of $\phi$, i.e., $\phi_{0}=\pi / 2$. 
[1] R. Crespo, R.C. Johnson, J.A. Tostevin, R.S. Mackintosh, and S.G. Cooper, Phys. Rev. C 49, 1091 (1994).

[2] K.M. Watson, Phys. Rev. 105, 1388 (1957); M.L. Goldberger and K.M. Watson, Collision Theory (John Wiley and Sons, New York, 1964).

[3] H.O. Meyer, P. Schwandt, G.L. Moake, and P.P. Singh, Phys. Rev. C 23, 116 (1981).

[4] L.R.B. Elton, Nucl. Phys. A89, 69 (1966).

[5] A.K. Kerman, H. McManus, and R.M. Thaler, Ann. Phys. (N.Y.) 8, 551 (1959).

[6] H.F. Arellano, F.A. Brieva, and W.G. Love, Phys. Rev. C 41, 2188 (1990); H.F. Arellano, W.G. Love, and F.A. Brieva, ibid. 43, 2734 (1991).

[7] Ch. Elster, T. Cheon, E.F. Redish, and P.C. Tandy, Phys. Rev. C 41, 814 (1990); C.R. Chinn, Ch. Elster, and R.M. Thaler, ibid. 44, 1569 (1991).

[8] R. Crespo, R.C. Johnson, and J.A. Tostevin, Phys. Rev. C 41, 2257 (1990).

[9] R. Crespo, R.C. Johnson, and J.A. Tostevin, Phys. Rev. C 48, 351 (1993).

[10] C.R. Chinn, Ch. Elster, and R.M. Thaler, Phys. Rev. C 48, 2956 (1993).

[11] R. Crespo, R.C. Johnson, and J.A. Tostevin, Phys. Rev. C 44, R1735 (1991); 46, 279 (1992).
[12] J. Hüfner and C. Mahaux, Ann. Phys. (N.Y.) 73, 299 (1972).

[13] H. Feshbach, Theoretical Nuclear Physics, Nuclear Reactions (John Wiley and Sons, New York, 1992), p. 383.

[14] R. Crespo, R.C. Johnson, and J.A. Tostevin, Phys. Rev. C 50, 2995 (1994).

[15] L.G. Arnold et al., Phys. Rev. C 25, 936 (1982).

[16] H. Horiuchi, Prog. Theor. Phys. 64, 184 (1980).

[17] E. Wigner, Phys. Rev. 40, 749 (1932).

[18] T.W. Donnelly and G.E. Walker, Phys. Rev. Lett. 22, 1121 (1969).

[19] R.C. Barrett and D.F. Jackson, Nuclear Sizes and Structure (Clarendon Press, Oxford, 1979).

[20] M. Lacombe, B. Loiseau, J.M. Richard, R. Vinh Mau, J. Côté, P. Pires, and R. de Tourreil, Phys. Rev. C 21, 861 (1980).

[21] E.F. Redish and K. Stricker-Bauer, Phys. Rev. C 36, 513 (1987).

[22] A.A. Ioannides and R.S. Mackintosh, Nucl. Phys. A467, 482 (1987); S.G. Cooper and R.S. Mackintosh, Inverse Problems 5, 707 (1989); S.G. Cooper and R.S. Mackintosh, computer code IMAGO, Open University Report No. OUPD9201, 1992 (unpublished). 